(C) 1996 IEEE. Personal use of this material is permitted. However, permission to reprint/republish this material

for advertising or promotional purposes or for creating new collective works for resale or redistribution to servers

or lists, or to reuse any copyrighted component of this work in other works must be obtained from the IEEE.

\title{
RESISTIVE-WALL INSTABILITY EXPERIMENT IN SPACE-CHARGE DOMINATED ELECTRON BEAMS*
}

\author{
H. Suk, J.G. Wang, and M. Reiser \\ Institute for Plasma Research, University of Maryland \\ College Park, MD 20742, USA
}

\begin{abstract}
The resistive-wall instability of charged particle beams has been experimentally studied in space-charge dominated electron beams. The beam energy is in the range of 3 to $8.5 \mathrm{keV}$, the beam current is between $25 \mathrm{~mA}$ and $62 \mathrm{~mA}$. In the experiment, electron beams produced from a thermionic cathode are passed through a $0.96 \mathrm{~m}$ long glass tube coated with a resistive material, and localized space-charge waves are launched in the beams. The ®rst preliminary experiment clearly shows the growth of slow spacecharge waves and the decay of fast space-charge waves. More systematic measurements and analysis of the growth(decay) rate are in progress.
\end{abstract}

\section{INTRODUCTION}

Charged particle beams with high brightness and low energy spread have many important applications such as high-current linear accelerators, free electron lasers, heavy-ion fusion drivers, etc. Such high quality beams should be accelerated and transported without serious beam quality deterioration due to instabilities and other effects. One potential problem is the resistivewall instability which causes an increase of the longitudinal beam energy spread. The increase of the longitudinal energy spread, in turn, may lead to transverse emittance growth due to coupling between the longitudinal and transverse forces acting on the particles.

Historically, the resistive-wall instability was $®$ rst studied by Birdsall et al. . ${ }^{[1],[2]}$ Their main interest was in the generation of microwaves. Later, Neil and Sessler ${ }^{[3]}$ studied the effect of the longitudinal instability on the relativistic beams in circulator accelerators. More recently, theoretical and simulation works for the longitudinal instability were done in connection with heavyion fusion induction linacs. ${ }^{[4]-[9]}$ However, a direct experimental veri®cation for the growth and decay rates of short perturbations has not been reported so far. Recently we have started a series of experiments to investigate the resistive-wall instability by launching localized space-charge waves in beams. The $®$ rst experiments show very clearly the growth of slow space-charge waves and the decay of fast space-charge waves.

In the following sections of this paper, these experiments on the resistive-wall instability are discussed.

\section{EXPERIMENTAL SETUP}

Figure 1 shows the experimental setup for the resistive-wall instability studies. As shown in the ®gure, the experimental system consists of the electron beam injector, the resistive-wall

\footnotetext{
*This work was supported by the U.S. Department of Energy
}

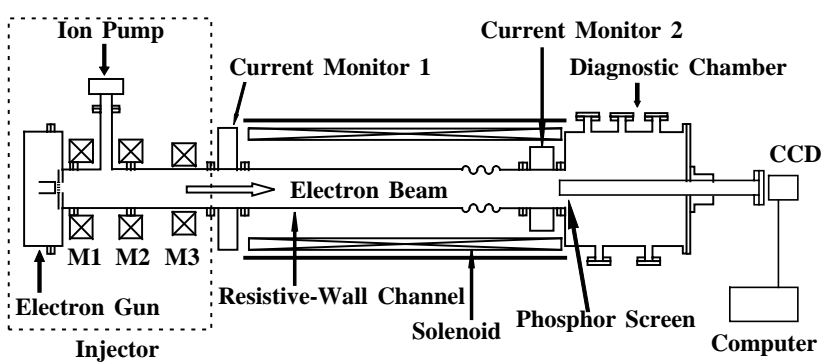

Figure. 1. Experimental setup.

channel, and the diagnostic chamber. The electron beam injector includes the variable-perveance gridded electron gun and the three matching lenses. The resistive-wall channel is a glass tube of which the inner surface is coated with $\mathrm{SnO}_{2}$. The length of the resistive part is $0.96 \mathrm{~m}$, the total resistance is $5.4 \mathrm{k} \Omega$, and the inner tube radius is $1.9 \mathrm{~cm}$. The beams from the electron gun are accelerated by high voltage $(3-8.5 \mathrm{keV})$, and then matched with the aid of the three solenoid lenses into the resistive transport channel. The beams inside of the resistive transport channel are uniformly focused by a long solenoid surrounding the glass tube. The magnetic ®eld of the solenoid is in the range of 46 to $66 \mathrm{G}$. The beam current signals at the entrance and exit of the channel are measured with two wall-current monitors which are $1.48 \mathrm{~m}$ apart from each other. The diagnostic chamber is placed at the end of the channel. An one-inch diameter phosphor screen in the diagnostic chamber can be alternatively inserted into the resistive transport channel for -uorescent beam image formation. The beam images from the phosphor screen are captured by the $\mathrm{CCD}$ (Charge Coupled Device) camera through a window and transferred to a computer for analysis. For studies of the resistive-wall effect on fast space-charge waves, beams with energies in the range of 6 to $8.5 \mathrm{keV}$ and currents in the range of 56 to $60 \mathrm{~mA}$ are used. The generalized beam perveance, $K=\left(I / I_{0}\right)\left(2 / \beta_{0}^{3} \gamma_{0}^{3}\right)$, is calculated to be between $1.1 \times 10^{-3}$ and $2.0 \times 10^{-3}$, with $I_{0}=1.7 \times 10^{4} \mathrm{~A}$ and $\beta_{0}, \gamma_{0}$ the usual relativistic velocity and energy factors. For instability studies of slow space-charge waves, beams with energies of 3 to $5 \mathrm{keV}$ and currents of 25 to $62 \mathrm{~mA}$ are used. In this case, the generalized beam perveance is about $2.9 \times 10^{-3}$. 

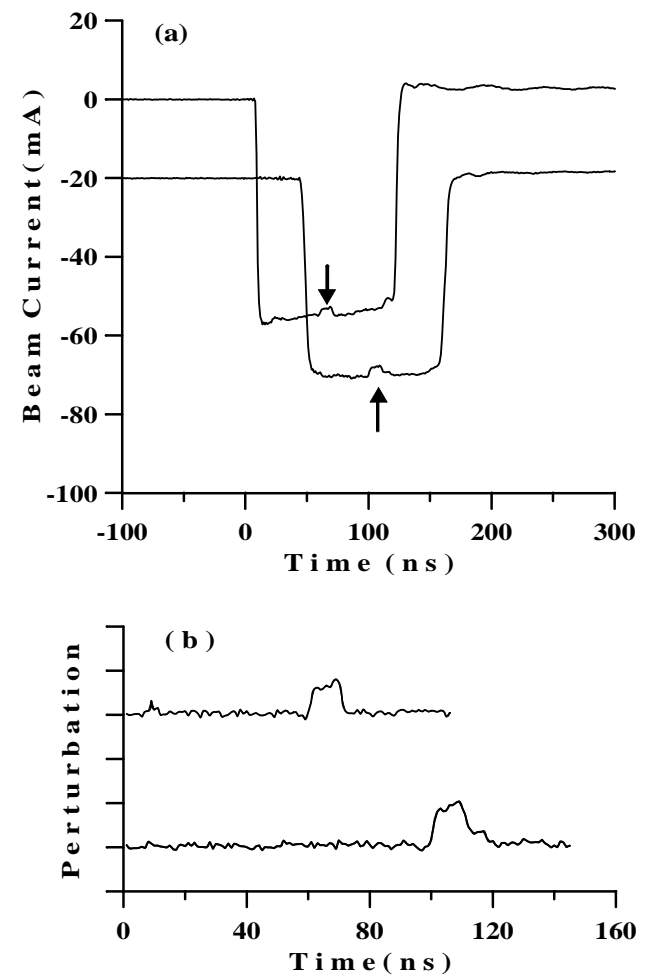

Figure. 2. (a) Typical current pro®les with a slow space-charge wave in the middle of the beam pulse. (b) Zoom-in view of the perturbation part of the beam pulses. The square-shaped slow wave with a width of about $10 \mathrm{~ns}$ is shown to grow. The vertical scale is $2.5 \mathrm{~mA} / \mathrm{div}$.

The beam pulses produced with a rectangular grid voltage pulse have a typical width of about $110 \mathrm{~ns}$. In order to launch localized space-charge waves, small square-shaped current perturbations with a width of about $10 \mathrm{~ns}$ are generated in the middle of these beam pulses. In the beam frame, the current perturbation can propagate in forward direction as a fast wave or it can propagate in backward direction as a slow wave. ${ }^{[10]}$ Generations of fast and slow waves depend on electron gun conditions such as anode-cathode distance, cathode temperature, cathodeanode voltage, etc. ${ }^{[1]}$ In some intermediate conditions, the perturbation can exist as a mixture of fast and slow waves. In our resistive-wall instability experiments, such mixed waves are avoided to abtain separate measurements of the behavior of individual fast and slow waves.

\section{RESULTS OF MEASUREMENTS}

A typical current pro®le with a slow space-charge wave is shown in Figure 2(a). The small square-shaped perturbation in the middle of the beam pulse, indicated by the arrow in the ${ }^{\circ} \mathrm{g}$ ure, moves in the bachward direction in the beam frame. Figure 2(b) shows the net perturbation signal produced by subtracting the beam pulse with the perturbation from the beam pulse without the perturbation. It is evident that the amplitude of the perturbation grows as it propagates in the beam.

Numerical calculations of the growth for slow space-charge waves were done for comparison with the experimental results.

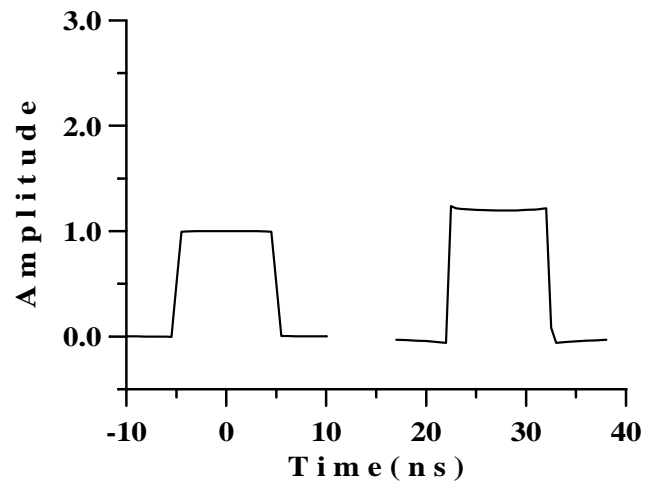

Figure. 3. Numerical result of the growth of a square-shaped perturbation. The perturbation is a slow wave in the beam with an energy of $4 \mathrm{keV}$ and a current of $48 \mathrm{~mA}$.

The calculation is based on the linear resistive-wall instability theory in the long-wavelength limit. ${ }^{[12]}$ In our experiment, the space-charge impedance is not much greater than the wall resistance of the transport channel. In this case, the growth rate is a function of frequency. The square-shaped perturbation in the beam contains a broad range of frequencies so that many frequency components of the perturbation should be taken into account. Figure 3 shows a numerical calculation result for a perturbation in the beam. In the ${ }^{\circledR}$ gure, the left signal is an initial perturbation at the location of the $®$ rst current monitor, and the right signal is a numerically calculated output at the location of the second current monitor. As shown in the ®gure, the amplitude of the perturbation increases and the perturbation is a little deformed due to the dispersion. In the calculation, the capacitive effect of the transport channel is not included, and a beam with an energy of $4 \mathrm{kV}$ and a current of $48 \mathrm{~mA}$ is used.

The experimental result for the growth rate of the slow spacecharge waves is shown in Figure 4. The round data points are from the experiment and the cross points are from numerical calculation. The triangular points are the spatial growth rates from the conventional long-wavelength limit formula ${ }^{[12]}$

$$
k_{i}=2 \pi \frac{R_{w}^{*}}{Z_{0}}\left(\frac{I}{I_{0}} \frac{1}{g \beta_{0} \gamma_{0}}\right)^{1 / 2},
$$

where $R_{w}^{*}$ is a wall-resistance per unit length, $Z_{0}$ a characteristic impedance of free space, $I$ the beam current, $I_{0}$ the characteristic current, $g$ the geometry factor. The formula is valid if the spacecharge impedance dominats over the wall impedance. As shown 


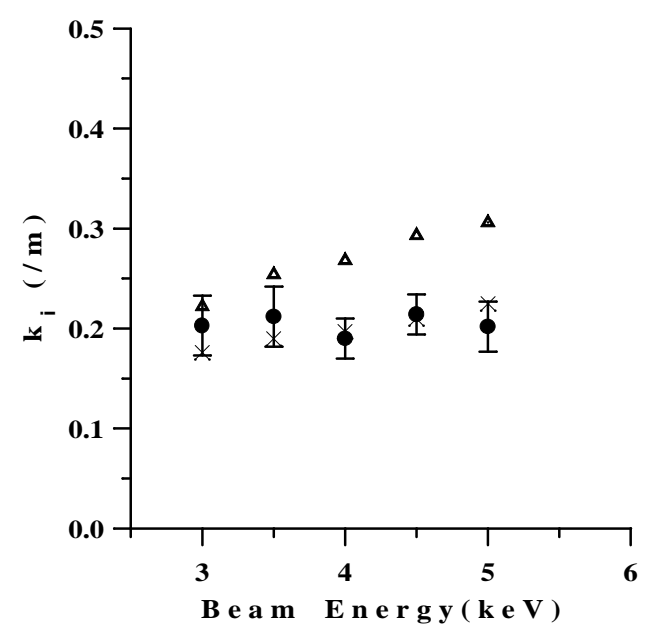

Figure. 4. Spatial growth rate of slow space-charge waves. The round data points are from the experiment, cross points are from numerical calculation, and the triangular points are from Eq. (1).

in the ${ }^{\circledR}$ gure, the experimental amplitude growth of the perturbation agrees with the numerical result.

In the experiment of fast space-charge waves, various beam energies between $6 \mathrm{keV}$ and $8.5 \mathrm{keV}$ are used. The experimental results of the spatial decay rates are shown in Figure 5(round data points). The cross points are from numerical calculations and the triangular points are from Eq. (1). As shown in the ${ }^{\circledR} g u r e$, the experimental data agrees with the numerical result. For analysis of localized space-charge waves in a resistive transport channel, it is necessary to take into account of wide range of frequencies in the localized perturbations.

\section{SUMMARY}

Localized slow space-charge waves are clearly observed to grow as they propagate in a transport channel with wallresistivity. The measured amplitude growth of the square-shaped perturbations is signi®cantly smaller than that of the long wavelength limit formula which applies to sinusoidal perturbations. Similarly the decay of localized fast space-charge waves are observed, and the measured amplitude decay is smaller than the formula for sinusoidal perturbations.

As pointed out in the introduction, the instability will cause beam quality deterioration. For better understanding and experimental veri ${ }^{\circledR}$ cation of beam quality deterioration, further experiments and analysis are needed. Our next experiments for timeresolved beam energy spread and emittance measurements will provide more information about the resistive-wall instability.

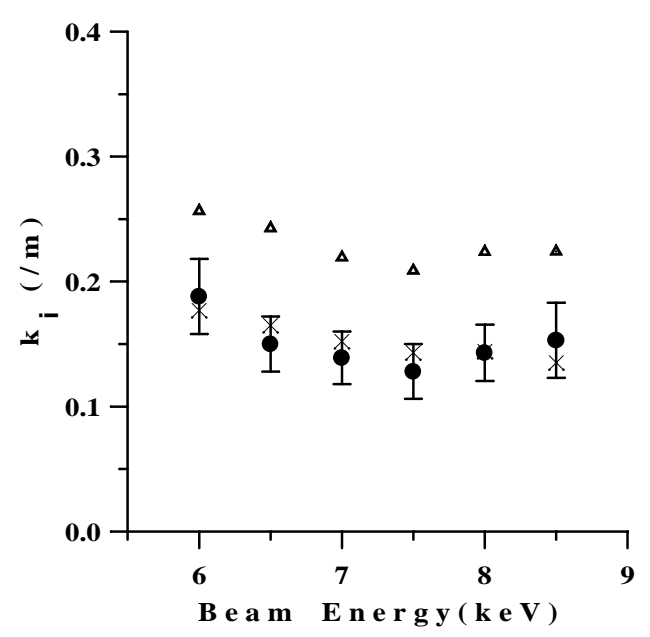

Figure. 5. Spatial growth rate of fast space-charge waves. The round data points are from the experiment, cross points are from numerical result, and the triangular points are from Eq. (1).

\section{References}

[1] C. K. Birdsall, G. R. Brewer, and A. V. Haeff, Proc. of I.R.E. 41, July 1953.

[2] C. K. Birdsall and J. Whinnery, J. Appl. Phys. 24, 314(1953).

[3] V. K. Neil and A. M. Sessler, Rev. Sci. Instr. 36, 429(1965).

[4] S. Humphries, Jr., J. Appl. Phys. 51, p. 2338, May 1980.

[5] E. Lee, Proc. of the 1981 Linear Accelerator Conference, Santa Fe, NM, October 19-23, 1981, pp. 263-265.

[6] J. Bisognano, I. Haber, L. Smith, and A. Sternlieb, IEEE Trans. on Nuclear Science, Vol. NS-28, No. 3, pp. 25132515, June 1981.

[7] J. G. Wang and M. Reiser, Phys. Fluids B5, 2286(1993).

[8] E. Lee, Nouvo Cimento 106A, 1679(1993).

[9] D. A. Callahan, A. B. Langdon, A. Friedman, and I. Haber, in the Proceedings of the 1993 Particle Accelerator Conference, Vol. 1, p. 730, Washington D.C., May 17-20, 1993.

[10] J. G. Wang and M. Reiser, in this proceedings.

[11] J. G. Wang, D. X. Wang, and M. Reiser, Phys. Rev. Lett. 71, 1836(1993).

[12] M. Reiser, Theory and Design of Charged Particle Beams(Wiley, New York, 1994), Sec. 6.3.2. 